\title{
« DEMAIN, UN JOUR NOUVEAU ? » UN RENVERSEMENT ÉLECTORAL CONFISQUÉ AU GABON
}

Alice Aterianus-Owanga, Mathilde Debain

Editions Karthala | « Politique africaine»

2016/4 $\mathrm{n}^{\circ} 144 \mid$ pages 157 à 179

ISSN 0244-7827

ISBN 9782811117986

Article disponible en ligne à l'adresse :

http://www.cairn.info/revue-politique-africaine-2016-4-page-157.htm

\section{Pour citer cet article :}

Alice Aterianus-Owanga, Mathilde Debain « «Demain, un jour nouveau ? » Un renversement électoral confisqué au Gabon », Politique africaine 2016/4 ( $\left.n^{\circ} 144\right)$, p. 157-179.

DOI 10.3917/polaf.144.0157

Distribution électronique Cairn.info pour Editions Karthala.

(C) Editions Karthala. Tous droits réservés pour tous pays.

La reproduction ou représentation de cet article, notamment par photocopie, n'est autorisée que dans les limites des conditions générales d'utilisation du site ou, le cas échéant, des conditions générales de la licence souscrite par votre établissement. Toute autre reproduction ou représentation, en tout ou partie, sous quelque forme et de quelque manière que ce soit, est interdite sauf accord préalable et écrit de l'éditeur, en dehors des cas prévus par la législation en vigueur en France. Il est précisé que son stockage dans une base de données est également interdit. 


\title{
CONJONCTURE
}

Alice Aterianus-Owanga et Mathilde Debain

\section{«DEMAIN, UN JOUR NOUVEAU? " UN RENVERSEMENT ÉLECTORAL CONFISQUÉ AU GABON}

\begin{abstract}
Sous la forme d'une chronique, cet article décrit la manière dont différents enjeux ont conduit, durant l'élection présidentielle gabonaise d'août 2016, à une mobilisation électorale en faveur de l'alternance, puis à sa confiscation. S'attachant à comprendre en quoi l'élection de 2016 s'est distinguée des précédentes, il insiste sur l'érosion des mécanismes de la fabrique du consentement et sur la formation d'une mobilisation contestataire multiforme, contrastant avec la logique de consentement passif qui était auparavant considérée comme l'un des traits des rapports de pouvoir au Gabon. Il démontre que si les possibilités de renversement réel du régime au pouvoir se sont avérées limitées, les élans portés par la société civile, par une opposition structurée et par une nouvelle génération de Gabonais ont permis, durant quelques mois, d'imaginer la possibilité d'un «jour nouveau», et $d^{\prime}$ inventer des espaces de protestation politique.
\end{abstract}

Mercredi 31 août 2016, sur le boulevard Triomphal de Libreville, à la tombée du jour. L'Assemblée nationale, ravagée par un incendie, laisse échapper d'amples volutes de fumée. Quatre jours après le scrutin, la victoire d'Ali Bongo Ondimba vient d'être annoncée par la Commission électorale nationale autonome et permanente (Cenap), à quelque 5000 voix d'écart par rapport à son principal rival, Jean Ping. Sans attendre, des manifestants descendent dans les rues pour protester contre une élection qu'ils considèrent truquée; alors que l'opposition était en tête avec 60000 voix d'avance et un taux de participation de $48 \%$ dans l'ensemble du pays, la province du Haut-Ogooué aurait, à la dernière minute, apporté la victoire au Président sortant, grâce à un taux de participation de $99 \%$, dont $95 \%$ des voix en faveur d'Ali Bongo. Dans la nuit du 31 août au $1^{\text {er }}$ septembre, les forces de l'ordre répriment violemment la contestation, entraînant plusieurs morts. Sur les antennes internationales, qui relaient les images d'un pays au «bord du chaos», la révolte en cours est expliquée, sur les ondes de RFI notamment, par une «tradition de défiance du peuple vis-à-vis des instances électorales», le scrutin reproduisant, selon ces lectures, un éternel processus de trucage et 
Conjoncture

de confiscation des élections, depuis le premier scrutin multipartite sous Omar Bongo en 1993 jusqu'à ceux de 2009 et de 2016.

Contre cette vision spasmodique et anhistorique de la contestation électorale, le présent article décrit, sous la forme d'une chronique des mois qui ont précédé l'élection et le scrutin lui-même, les différentes forces et les enjeux spécifiques qui ont conduit à la mobilisation électorale du 27 août 2016 en faveur de l'alternance, puis à sa confiscation. Il cherche en particulier à comprendre comment le Gabon, souvent décrit comme un pays stable, retranché derrière la logique $d u$ «on va encore faire comment ? $^{1}$ » et $d u$ consentement passif des populations, est devenu, en quelques mois, le site d'une protestation politique qu'il n'avait pas connue depuis les événements de 19932. À cette date en effet, la victoire d'Omar Bongo par 51,20\% des suffrages avait entraîné de violents affrontements entre les forces de l'ordre et les manifestants qui défendaient la victoire de Paul Mba Abessole, candidat du Rassemblement national des bûcherons. Le retour à l'ordre, négocié avec le concours de la France lors des Accords de Paris, avait conduit à l'entrée au gouvernement du principal opposant.

À partir du cas gabonais, cet article entend contribuer à une meilleure compréhension de la légitimation et de la contestation du pouvoir en Afrique centrale, une région fréquemment décrite comme le «cœur des ténèbres » politiques du continent, gelée dans une ère sans fin de régimes autocratiques. Il s'attache à comprendre en quoi l'élection de 2016 s'est distinguée des précédentes, et comment l'espoir d'un «jour nouveau ${ }^{3}$ » a émergé chez une partie de la population gabonaise. Il insiste sur l'érosion des mécanismes de la fabrique du consentement et sur la formation multi-sites d'une mobilisation en faveur de l'alternance politique, portée à la fois par des acteurs politiques, des membres de la société civile et des artistes de hip-hop, agents clés de la politisation des jeunes au Gabon ${ }^{4}$.

1. Cette expression courante au Gabon exprime la résignation et le sentiment d'impuissance des Gabonais face à des mécanismes politiques, sociaux ou économiques immuables. Voir aussi à ce propos F. Bernault et J. Tonda, «Le Gabon: une dystopie tropicale», Politique africaine, n 115, 2009, p. 6-26.

2. En 2009 également, des violences avaient éclaté à Port-Gentil suite à l'élection d'Ali Bongo, mais leur ampleur avait été bien moindre.

3. Ce titre, qui fait référence à un célèbre film gabonais réalisé par Pierre-Marie Ndong à la fin des années 1970, a été repris comme slogan par des partisans de l'alternance durant l'élection.

4. Les acteurs du monde du rap deviennent, depuis un certain nombre d'années, des leaders d'opinion importants pour la jeunesse, dans un pays composé en grande majorité de jeunes de moins de 30 ans (64\% de la population). 
«Demain, un jour nouveau? » Un renversement électoral confisqué au Gabon

\section{UN SEPTENNAT DE MONTÉE EN CONTESTATION}

En juillet 2009, Ali Bongo est désigné candidat du Parti démocratique gabonais (PDG) pour l'élection présidentielle à venir en août 2009, un mois après le décès de son père Omar Bongo, qui avait présidé le Gabon depuis 1967. Entendant rompre avec une image de perpétuation dynastique du pouvoir, Ali Bongo se présente comme le candidat des jeunes et du changement. Il s'entoure d'une équipe de rappeurs qu'il met au centre de sa communication et de ses meetings ${ }^{5}$. Ils réalisent pour lui la chanson de campagne intitulée «On te suit», un appel à «suivre» le candidat défenseur des jeunes, des femmes, des étudiants, et de tous les Gabonais. Avec son projet d'« Avenir en confiance», Ali Bongo acquiert le soutien d'une partie des jeunes et des artistes, leur assurant un renforcement des structures démocratiques, davantage de lutte contre les inégalités, la construction d'établissements scolaires et d'hôpitaux, et un appui actif aux artistes, par la mise en place d'un système de droits d'auteur. Au terme du scrutin, tenu le 30 août 2009, Ali Bongo est proclamé vainqueur de l'élection présidentielle par la commission électorale. Malgré les contestations de l'opposition et les violences qui éclatent dans la capitale économique Port-Gentil, il célèbre son investiture le 16 octobre 2009 par un grand concert au Jardin botanique, accompagné de ses supporters rappeurs.

Dès son élection, Ali Bongo met en place un programme d'«émergence», organisé autour de trois pôles : le Gabon industriel, le Gabon vert et le Gabon des services. Pour contourner l'immobilisme de l'administration, il décide de gouverner par agences placées sous la tutelle directe de la présidence de la République. On voit ainsi naître l'agence nationale des grands travaux, l'agence nationale de l'Habitat, l'agence nationale des parcs du Gabon, ou l'agence des Bourses et stages, entièrement soustraites au contrôle budgétaire du parlement. Parallèlement, des pratiques d'octroi des marchés publics à des entreprises privées appartenant au Président et ses proches sont très vite révélées ${ }^{6}$, et les continuités avec le système de prédation

\footnotetext{
5. Jadis artiste dans les années 1970, Ali Bongo exécuta lui-même une performance rappée sur scène, face à un millier de jeunes. Lire A. Aterianus-Owanga, «Les pratiques musicales urbaines: des nouvelles voix(es) démocratiques au Gabon?", Émulations. Revue des jeunes chercheurs en sciences sociales, $n^{\circ}$ 9, 2011, <www.revue-emulations.net/archives/n9/aterianus>, consulté le 6 décembre 2016.

6. Déjà pour l'année 2012, l'économiste Mays Mouissi note que les marchés liés aux travaux publics sont conclus, dans plus de 50\% des cas, au travers d'ententes directes, de gré à gré, en dehors de tout appel d'offres. Plus tard, en avril 2015, le site Mediapart révélait par exemple le scandale de Delta Synergie, une holding privée appartenant à Ali Bongo, à sa sœur Pascaline et à différents proches de la famille Bongo, qui détient des parts dans une cinquantaine des plus importantes entreprises du Gabon. Voir M Mouissi, «Gabon: 1'ANGT et le scandale des marchés publics par
} 
Conjoncture

des ressources publiques développé sous Omar Bongo sont mises en évidence?.

Rapidement, «l'émergence» devient l'objet d'une défiance manifeste de la part des populations. En effet, le septennat est marqué par une profusion de dépenses ostentatoires de la famille présidentielle ${ }^{8}$ et d'événements festifs aux budgets onéreux ${ }^{9}$, inversement proportionnels à la déficience croissante des institutions de santé, d'enseignement ou des services en eau et en électricité. Dans le registre de la rumeur comme dans la presse d'opposition, les dépenses de l'État et les choix politiques du Président commencent à faire l'objet d'un mécontentement manifeste; «l'émergence» se convertit dans les discours populaires en «immergence» ou en «énervance». En outre, la répression violente des tentatives de contestation populaire et l'absence de dialogue entre Ali Bongo et les acteurs de l'opposition font éclater le consentement qu'Omar Bongo avait négocié auprès de ses opposants durant ses dernières années de pouvoir. L'assujettissement de la classe politique reposait alors sur l'étouffement des velléités contestataires par le dialogue, la remise de contreparties matérielles ou financières, et la domination de la sphère religieuse ${ }^{10}$, autant d'outils de fabrique du consentement qu'Ali Bongo peine à manipuler.

Durant le septennat, plusieurs événements cristallisent les tensions autour des modes de gouvernement du nouveau Président. Le premier survient au moment de l'arrivée au pouvoir d'Ali Bongo, en septembre 2009, lors de la répression des émeutes de Port-Gentil. Les morts passés sous silence et la censure implicite qui entoure l'événement nourrissent un premier motif de rancœur. À ces violences inaugurales de «l'émergence», s'ajoute celle des crimes rituels, qui rencontrent une médiatisation croissante dans les premières années du septennat ${ }^{11}$. Ils deviennent l'objet principal des discussions dans l'espace public et le motif d'une hantise chez les Gabonais qui accusent les

entente directe» [en ligne], mays-mouissi.com, 26 avril 2016, <www.mays-mouissi.com/2016/04/26/ gabon-langt-scandale-marches-publics-entente-directe/>, consulté le 26 décembre 2016.

7. Voir P. Moundounga Mouity et E. Nguema-Minko, «Présentation», in P. Moundounga Mouity et K.-F. Ndjimba (dir.) Le Gabon à l'épreuve de la politique de l'émergence. Diagnostic et pronostic, SaintDenis, Publibook, 2012, p. 23.

8. Le parc automobile du Président ou les dépenses colossales de sa sœur Pascaline en billets d'avion et en produits de luxe ont été épinglés par plusieurs médias.

9. On citera notamment le concert du groupe de rap français, Sexion d'Assaut, en juin 2013, la tenue de plusieurs éditions du «Moto-show», le carnaval brésilien de février 2013, ou encore la Coupe d'Afrique des nations organisée entre le Gabon et la Guinée équatoriale en janvier-février 2012.

10. F. Ngolet, «Ideological Manipulations and Political Longevity: the Power of Omar Bongo in Gabon since $1967 »$, African Studies Review, vol. 43, n² 2, 2000, p. 55-71.

11. Au-delà de la presse locale, des articles parus dans Jeune Afrique et le court reportage réalisé par la chaîne française Canal+ ont alarmé la communauté internationale sur ce phénomène. 
dirigeants d'être les commanditaires de trafic d'organes à des fins de fétichisme et de maintien au pouvoir ${ }^{12}$. Liée à un imaginaire local qui rattache le pouvoir politique à l'extraction des forces vitales des citoyens ${ }^{13}$, cette explosion du sentiment d'insécurité se manifeste par différentes mobilisations en apparence «apolitiques», mais fortement hostiles aux dirigeants, comme une grande marche de protestation contre les crimes rituels, organisée en avril 2013 à Libreville et dans la diaspora ${ }^{14}$, ou, quelques mois plus tard, l'appel au boycott d'un grand spectacle hip-hop organisé par une agence proche de la famille présidentielle ${ }^{15}$. Une partie de la population librevilloise considère en effet la manifestation comme un guet-apens organisé pour attirer les jeunes dans un quartier périphérique de Libreville à des heures tardives, sans considération pour les risques d'enlèvement.

Enfin, les critiques de l'opinion publique se cristallisent dans les dernières années du mandat d'Ali Bongo sur deux éléments, alimentés par la publication du livre de Pierre Péan en novembre $2014^{16}$. Le premier porte sur la nationalité d'Ali Bongo Ondimba, soupçonné d'être un orphelin nigérian adopté par Omar Bongo lors du conflit biafrais. La validité de son acte de naissance, un acte français dont l'original serait conservé aux archives de Nantes, est au centre des débats. Le second élément a trait à l'omniprésence de son directeur de cabinet, Maixent Accombressi, citoyen d'origine béninoise naturalisé Gabonais, considéré comme la pièce maîtresse du premier cercle présidentiel et de la captation des ressources de l'État. Ces deux crispations, manifestes dans les discours populaires qui précèdent la campagne électorale de 2016, ont fréquemment conduit les journalistes à parler d'une «xénophobie gabonaise» et d'un rejet des étrangers au Gabon. Mais comme l'ont déjà montré d'autres travaux sur les poussées xénophobes dans les villes africaines ${ }^{17}$, l'enjeu ici est avant tout économique et politique: la parole xénophobe constitue le relais de la déroute de la population face au creusement des inégalités socio-économiques et face à une gouvernance peu encline au dialogue.

12. A. Mary et M. Mebiame-Zomo, «Épidémie de "crimes rituels" au Gabon. Des affaires de sorcellerie au scandale de l'impunité», in S. Fancello (dir.), Penser la sorcellerie en Afrique, Paris, Hermann, 2015.

13. J. Tonda, Le Souverain moderne: le corps du pouvoir en Afrique centrale (Congo, Gabon), Paris, Karthala, 2005.

14. Cette marche est d'abord organisée sous l'impulsion de la société civile, puis reprise par la première dame, Sylvia Bongo Ondimba.

15. Ces données sont extraites d'observations ethnographiques effectuées à Libreville, où l'une des auteures de l'article résidait durant toute l'année 2013 (et auparavant).

16. P. Péan, Nouvelles affaires africaines. Mensonges et pillages au Gabon, Paris, Fayard, 2014.

17. A. Wa Kabwe-Segatti, «Violences xénophobes en Afrique du Sud: retour sur un désastre annoncé», Politique africaine, $n^{\circ} 112,2008$, p. 99-118. 
Conjoncture

Résultat de ces différents motifs de colère, de nombreuses grèves aboutissent en 2015 au blocage général de l'administration publique. Ces protestations, souvent présentées comme une conséquence de la chute brutale du prix du baril de pétrole (à partir du mois de juin 2014) et du tarissement des sources de financement de la domination, existaient pourtant préalablement à la crise pétrolière. En réalité, la grève illimitée qui touche tous les secteurs de l'administration fait suite à plusieurs mouvements de grogne syndicale dans les années précédentes. Elle est, avant tout, l'expression d'un mécontentement général face à l'absence de dialogue politique ou de mesures contre l'accroissement des inégalités, des problématiques qui précèdent la chute des prix du pétrole et soulignent, bien avant l'élection, l'échec du programme $\mathrm{d}^{\prime}$ «émergence».

Les événements qui surviennent dans la société civile et le monde artistique en 2016 soulignent combien l'enjeu devient désormais la critique d'un régime prédateur engagé dans une entreprise de restauration autoritaire, à rebours de l'image ancienne attribuée au Gabon de «dictature soft 18 ».

\section{DES VOIX DISSIDENTES DANS LA SOCIÉTÉ CIVILE}

Dès le début de l'année 2016, différentes voix dissidentes se font entendre du côté de la société civile, tantôt en affirmant une neutralité politique, tantôt en appelant à la création d'une alternance démocratique et à la chute du système Bongo. Parmi elles, Marc Ona Essangui occupe depuis plusieurs années l'espace laissé vacant par l'opposition divisée. Fondateur de l'ONG environnementale Brainforest, il s'implique dans la lutte contre les malversations financières et, en 2016, il dénonce l'appropriation des marchés de l'État par les proches d'Ali Bongo, appelant à la fédération d'une opposition solide $^{19}$. De même, dans la diaspora, des intellectuels publient des ouvrages et des articles critiquant les échecs de l'émergence, à l'instar de Laurence Ndong, qui organise plusieurs conférences de presse en France pour présenter son livre, Gabon, pourquoi j'accuse... ${ }^{20}$, ou du blogueur Mays Mouissi, dont

18. F. Bernault, «Gabon. Les paradoxes d'une dictature "soft" » [en ligne], Le Monde, 5 septembre 2016, <www.lemonde.fr/afrique/reactions/2016/09/05/gabon-les-paradoxes-d-une-dictaturesoft_4992691_3212.html>, consulté le 6 décembre 2016.

19. D. MBaye, «Interview Marc Ona Essangui : “Ali Bongo ne va pas rempiler” » [en ligne], Les Afriques, 24 mars 2016, <www.lesafriques.com/actualite/interview-marc-ona-essanguiali-bongo-ne-va-pas-rempiler.html?Itemid=89>, consulté le 6 décembre 2016.

20. L. Ndong, Gabon, pourquoi j'accuse..., Paris, l'Harmattan, 2016. 
les analyses économiques s'emploient à mettre en lumière les dysfonctionnements de la gestion de l'État ${ }^{21}$.

Au-delà de ces sphères intellectuelles, de nouveaux mouvements sociaux émergent aussi dans les quartiers de Libreville. Au début de l'année 2016, un collectif dénommé la Voix du mapane organise par exemple des chantiers de solidarité à l'égard des classes modestes et des actions de protestation dans Libreville, condamnant aussi bien la corruption de l'État que la désorganisation de l'opposition. Vêtus de treillis kaki, ses membres se présentent comme «un groupe de pression politique» composé des jeunes des quartiers défavorisés, dont quelques célébrités du monde du hip-hop.

Sur le plan syndical, la confédération Dynamique unitaire poursuit son mouvement de grève illimitée dans l'administration ${ }^{22}$ et fait entendre son désir d'alternance dans ses assemblées générales et lors de ses interventions dans les médias. Le 25 juin 2016, plusieurs associations et personnalités (Marc Ona Essangui, la Voix du mapane, Dynamique unitaire, Femmes lèvetoi) se réunissent autour d'un carrefour central de Libreville, le rond-point de la Démocratie ${ }^{23}$, avec un mot d'ordre explicite: disqualifier la candidature d'Ali Bongo et agir pour la chute du régime en place.

Parallèlement, d'autres appels au vote et à l'alternance exercent un impact sur la mobilisation des Gabonais durant cette élection, dont les chansons de rap, très populaires auprès des jeunes ${ }^{24}$. Après une période de relative atonie de la scène contestataire, consécutive à l'élection de 2009 et au raffermissement des techniques de censure ou de cooptation, la fin du septennat accueille une explosion de critiques de «l'émergence», aussi bien par des rappeurs critiques que par les anciens supporters d'Ali Bongo. Le rappeur Keurtyce E, connu dans sa génération pour sa virulence, est le premier à publier la chanson la plus ouvertement engagée contre le régime en place, en décembre 2015. Intitulé «On va tourner la page», ce titre s'adresse à Ali Bongo sur le ton de

21. Voir les articles consacrés à l'économie gabonaise sur le site Internet de Mays Mouissi, et notamment M. Mouissi, «Gabon: Ces dépenses atypiques financées sur fonds publics » [en ligne], mays-mouissi.com, 24 mai 2016, <www.mays-mouissi.com/2016/05/24/gabon-depenses-atypiquesfinancees-fonds-publics/>, consulté le 6 décembre 2016.

22. Sans qu'on connaisse exactement le taux de participation à ces grèves, seule la menace de suspension des salaires amènera à une reprise des cours à partir de mars 2015 dans les écoles gabonaises.

23. Ce rond-point est ainsi nommé car il jouxte la vaste structure qui avait été construite en 1977 pour accueillir l'OUA, la «cité de la démocratie». Du fait de sa position centrale, il est fréquemment employé comme lieu de manifestation.

24. Les musiques hip-hop (rap, R\&B, afro-rap) sont en effet devenues extrêmement populaires depuis le milieu des années 1990. À ce propos voir notamment A. Aterianus-Owanga, «"Gaboma”, "Kainfri" et "Afropéen". Circulation, création et transformation des catégories identitaires dans le hip-hop gabonais », Cahiers d'études africaines, n²16, 2014, p. 945-974. 
Conjoncture

la menace, l'incitant à quitter le palais présidentiel et il annonce la préparation d'un «coup d'État électoral ${ }^{25}$ », désignant ainsi les manipulations pressenties de la Cenap et de la cour constitutionnelle par l'exécutif. Quelques semaines plus tard, c'est depuis la diaspora qu'un autre rappeur fait sensation, avec un titre qui ne tarde pas à enflammer la toile et à devenir la bande-son des meetings de l'opposition, «Mister zéro 26 ». Revenant sur les promesses non tenues de l'ancien candidat, le rappeur Saik'1ry démonte les sept années de gouvernance du Président sortant, et clame la nullité du bilan de «Mister zéro ». D'autres morceaux suivent, produits par des rappeurs du Gabon et de la diaspora, qui se veulent autant d'expressions de la non-adhésion des jeunes au projet de société d'Ali Bongo et d'incitations à se mobiliser contre le Président sortant.

En 2016, comme cela avait été le cas en 2009, les chansons de rap servent de réservoir d'inspiration des slogans de campagne et d'outils d'attraction des foules, le langage populaire des jeunes se voyant mis au service des programmes électoraux. Tandis qu'Ali Bongo fait un remix des chansons utilisées sept ans plus tôt, le camp de l'opposition réunit les foules autour du titre «Mister zéro » et d'un son produit pour l'élection : «Jean Ping, c'est dosé». Ce dernier reprend l'expression polysémique «c'est dosé», qui traduit, dans l'argot des jeunes, un fait accompli proprement, justement ou de façon mesurée. Le titre assure qu'avec le candidat de l'opposition, les promesses seront tenues et que les hôpitaux, les écoles, les routes et la démocratie seront développés en quantité.

Mais à la différence de 2009, nombre d'artistes qui avaient soutenu plus ou moins activement le candidat de «l'émergence» s'engagent cette fois contre Ali Bongo, dénonçant dans plusieurs chansons le «coup d'État électoral» en préparation, ou abordant des sujets habituellement objets d'une censure voilée, comme celui de la nationalité du Président. Ce revirement des ex-supporters d'Ali Bongo témoigne d'un rejet de la politique menée durant le septennat, rejet qui va s'amplifier à la veille de l'élection suite au tournant répressif pris par le parti au pouvoir à l'encontre de l'opposition et de la société civile. L'usage de techniques coercitives de gouvernement se banalise en effet durant cette période et la fréquence des arrestations arbitraires donne à la précampagne des allures de «chasse à l'homme».

Le 9 juillet 2016, des associations de la société civile appellent à la mobilisation du peuple lors d'une marche pacifique au rond-point de la Démocratie,

25. Keurtyce E, «On va tourner la page» [en ligne], YouTube, 2015, <www.youtube.com/watch?v= Xegaw8aX3B4>, consulté le 6 décembre 2016.

26. Saik'1ry, «Mister zéro a.k.a Ali Bongo» [en ligne], Soundcloud, 2016, <soundcloud.com/rap-gabonais/saik1ry-mister-zero-aka-ali-bongo>, consulté le 6 décembre 2016. 
pour protester contre le dépôt de candidature d'Ali Bongo, déjà accusé de fomenter un «coup d'État électoral». La police charge et tire des bombes lacrymogènes. La manifestation est avortée et aboutit à l'arrestation de vingt personnes de la société civile. Parmi eux se trouvent Jean-Remy Yama, président de la confédération syndicale Dynamique unitaire, des proches de l'opposition et des leaders étudiants. Plusieurs membres du mouvement la Voix du mapane, dont le célèbre rappeur Buung Pinz, sont par la suite activement recherchés, et une traque débute dans les rues de Libreville. Le 14 juillet, au domicile de Buung Pinz, deux de ses frères sont arrêtés, dont son cadet Lörd Helkaass, rappeur connu pour son absence d'engagement politique et ses collaborations avec une radio proche du parti au pouvoir. Dès l'annonce de cette arrestation, la communauté hip-hop réagit unanimement, y compris les médias et partisans du candidat Ali Bongo (comme un de ses chargés de mission et porte-parole), pour dénoncer une arrestation arbitraire ${ }^{27}$. À partir de cette date, plusieurs personnalités qui avaient autrefois collaboré avec les équipes de campagne d'Ali Bongo vont afficher une distance plus nette vis-à-vis du régime. D'autres, à l'inverse, comme les rappeurs impliqués dans la campagne du PDG, accentuent leur promotion du président-candidat, et la violence des échanges entre les partisans et détracteurs du PDG s'accentue.

Finalement, l'une des arrestations les plus spectaculaires survient à la veille de l'ouverture de la campagne et touche cette fois un activiste connu des réseaux sociaux, résidant aux États-Unis : Landry Amy Washington. Le jeudi 10 août, à son arrivée à l'aéroport de Libreville, il est interpellé et incarcéré au motif $\mathrm{d}^{\prime}$ «injures au Président ${ }^{28}$ ». Malgré les appels de la société civile, la mobilisation des avocats du barreau de Paris, et quelques articles de presse internationale dénonçant ces arrestations arbitraires, Landry Amy Washington, Jean-Remy Yama, et d'autres personnalités resteront emprisonnés pendant toute la période de l'élection ${ }^{29}$.

27. Ce dernier est finalement relâché deux jours plus tard, après plusieurs heures d'interrogatoire.

28. Landry Amy Washington est devenu célèbre depuis plusieurs années pour les vidéos qu'il publie sur YouTube, où il critique la politique et la personnalité d'Ali Bongo, reprenant parfois des rumeurs homophobes sur la supposée sexualité du président. Voir par exemple L. Ami, «Landry confirme les propos de Lanlaire. Ali Bongo est homosexuel» [en ligne], YouTube, 11 février 2015, $<$ www.youtube.com/watch?v=MdPjGx9xAYA>, consulté le 25 décembre 2016. Sur les rapports entre la parole homophobe et le politique au Gabon, voir A. Aterianus-Owanga, " "L'émergence n'aime pas les femmes!" Hétérosexisme, rumeurs et imaginaires du pouvoir dans le rap gabonais ", Politique africaine, $\mathrm{n}^{\circ} 126,2012, \mathrm{p} .49-68$.

29. Jean-Remy Yama a été libéré le 6 octobre 2016 (après trois mois d'incarcération), le leader étudiant Firmin Ollo le 14 décembre 2016 (après quatre mois de détention), et au moment de la finalisation de cet article, Landry Amy Washington était toujours en détention, de même que des personnalités de l'opposition. 
Conjoncture

En parallèle de la société civile, le monde politique est lui aussi bouleversé en 2016 par différents mouvements, signes d'une mobilisation qui avait tardé à s'organiser durant le septennat, mais qui prend forme à l'orée des élections.

\section{PRÉCAMPAGNe DANS LES DEUX CAMPS}

Le 12 mars 2016, lors de son 48 e anniversaire, le PDG investit Ali Bongo comme candidat à la présidentielle. Ce dernier annonce les contours de son programme de campagne sur «l'égalité des chances». Le meeting revêt d'autant plus d'importance que le PDG est alors en proie à une dissidence interne et à une vague de démissions sans précédent. Dès le mois de janvier, l'ancien président de la Commission de l'Union africaine, Jean Ping, annonce sa candidature à l'élection présidentielle et dénonce dans les médias la situation du Gabon, "une dictature pure et simple entre les mains d'une famille, d'un clan ${ }^{30}$ ». Ancien directeur de cabinet et longtemps ministre d'Omar Bongo, il est choisi pour représenter le Front de l'opposition pour l'alternance (Fopa) le 15 janvier 2016, sans avoir eu d'affiliation partisane déclarée depuis son départ du PDG en février 2014.

Après les démissions de Jacques Adiahénot en 2014 puis celle de René Ndemezo Obiang en 2015, autres anciens caciques du régime de Bongo père, une série de députés et de personnalités du PDG donnent leur démission le 10 mars 2016 et créent un parti dissident nommé PDG Héritage et modernité. Ils expliquent eux aussi réagir contre l'absence de « démocratie dans le PDG ${ }^{31}$ », et notamment contre la proclamation d'Ali Bongo comme candidat à la présidentielle, sans que le parti n'ait procédé à des primaires. Finalement, quelques semaines plus tard, le vendredi $1^{\mathrm{er}}$ avril, neuf députés et le président de l'Assemblée nationale, Guy Nzouba Ndama, annoncent à leur tour leur départ du PDG au cours de séances plénières. Au terme de cette vague de démissions, Guy Nzouba Ndama est investi candidat à l'élection présidentielle du Rassemblement Héritage et modernité, tandis que l'Union nationale, considérée comme le principal parti de l'opposition depuis 2009, soutient la candidature de Casimir Oyé Mba, ancien Premier ministre d'Omar Bongo

30. «Entretien avec Jean Ping: “Le Gabon est une dictature pure et simple entre les mains d'une famille, d'un clan" ", Le Monde, 2 mars 2016, <www.lemonde.fr/afrique/article/2016/03/02/jeanping-le-gabon-est-une-dictature-pure-et-simple-entre-les-mains-d-une-famille-d-un-clan_4874874_ 3212.html\#HvuLiW4TVOo4dZFS.99>, consulté le 6 décembre 2016.

31. Voir C. N. Nkany, «Implosion du PDG: “Héritage et Modernité,, la nouvelle aile du parti?» [en ligne], Gaboneco, 11 mars 2016, <www.gaboneco.com/implosion-du-pdg-heritage-et-modernitela-nouvelle-aile-du-parti.html $>$, consulté le 6 décembre 2016. 
«Demain, un jour nouveau? "Un renversement électoral confisqué au Gabon

qui avait rejoint l'opposition en 2009. Cette vague de candidatures dissidentes fragilise le parti au pouvoir mais également l'opposition, critiquée pour son incapacité à fédérer une véritable alliance ou à énoncer un programme politique solide, en dehors de la polémique sur la nationalité du Président.

Cette polémique accompagne le dépôt des candidatures à l'élection présidentielle début juillet 2016 et elle mobilise particulièrement l'attention des médias. Plusieurs candidats de l'opposition intentent un recours pour démontrer l'inéligibilité du candidat Ali Bongo, accusé d'être de nationalité nigériane et d'employer, dans son dossier de candidature, un faux acte de naissance. Lors de la délibération du bureau de la Cenap, sur les 18 candidatures déposées, seule celle d'Ali Bongo Ondimba ne fait pas consensus. Elle est néanmoins validée par une majorité de cinq membres contre trois. Cette validation non consensuelle constitue pour l'opposition et la société civile une première preuve de la partialité de la Cenap, de même que le rejet du recours déposé ensuite par les opposants à la Cour constitutionnelle. Finalement, 14 candidatures sont validées le 14 juillet 2016.

Avant le lancement officiel de la campagne, de nouveaux événements viennent bousculer le champ politique, dévoilant un peu plus la fragilité d'Ali Bongo à l'intérieur du PDG et l'érosion de ses modes de domination. Le 24 juillet 2016, lors de sa tournée à l'intérieur du Gabon, le Président fait escale dans la province du Woleu-Ntem où son discours doit être introduit par le député de la localité. Au lieu d'un discours d'accueil, le député Bertrand Zibi Abeghé annonce en direct qu'il quitte le PDG et son poste de député, sous les acclamations de la population. Il justifie son acte en déplorant l'état de misère dans laquelle vivent les habitants de sa circonscription et la détérioration de leurs conditions de vie. Ce retrait d'un proche du système vient affaiblir encore davantage un PDG en perte de vitesse, et diminue les rangs des soutiens du Président.

Malgré ces revirements dans le parti au pouvoir, la campagne électorale débute le 13 août 2016 avec des déséquilibres matériels et financiers considérables en faveur du pouvoir, dans un premier temps seulement.

\section{ÉCONOMIES DU SPECTACLE ET INVERSION DE LA CAMPAGNE}

Le matin du samedi 13 août 2016, Libreville se réveille sous de nouvelles couleurs. Dans la nuit, la ville a été recouverte d'affiches à l'effigie du Président sortant. L'après-midi du même jour, celui-ci ouvre sa campagne par un meeting et un concert dans le stade de l'Amitié sino-gabonaise d'Angondjé, accompagné d'un nombre important d'artistes populaires. Des bus de la société nationale Sogatra et des minibus sont mis à disposition pour assurer 
Conjoncture

la présence des populations. Quelques jours plus tard, dans les médias nationaux et sur les réseaux sociaux, on annonce une participation de 40000 personnes au meeting, 60000 d'après les instances du PDG. La fête est décrite comme «impeccable», mais des journalistes critiquent les points aveugles du discours d'Ali Bongo, notamment sur la question de la révision de la constitution concernant la durée et le nombre de mandats ${ }^{32}$, à laquelle se sont engagés la plupart des autres candidats ${ }^{33}$. Pour les dissidents, notamment sur les réseaux sociaux, il ne fait nul doute que les foules réunies répondent présentes pour bénéficier de récompenses financières, s'élevant selon les rumeurs entre 5000 et 40000 francs CFA. Les gadgets de campagne distribués incluent les classiques tee-shirts à l'effigie du candidat, mais aussi des montres, des sacs de riz, des denrées alimentaires de première nécessité, des congélateurs, des tronçonneuses, des smartphones et des tablettes tactiles ${ }^{34}$.

Dans le camp de l'opposition, Jean Ping inaugure sa campagne dans la ville de Lambaréné, au centre du pays, tandis que Guy Nzouba Ndama est contraint d'annuler le lancement de la sienne, après s'être vu refuser l'autorisation d'accès au stade de Nzeng-Ayong par l'Agence nationale de gestion des infrastructures sportives et culturelles. Cet accaparement des moyens de l'État, des lieux de rassemblement et des panneaux d'affichage publicitaire par le parti au pouvoir se poursuivra durant l'ensemble de la campagne, au détriment des autres leaders politiques. Les moyens de communication du Président sortant lui permettent ainsi d'organiser une tournée dans l'intérieur du Gabon agrémentée de spectacles de grande envergure.

Le désavantage matériel de l'opposition est toutefois contrebalancé par plusieurs événements, qui font progressivement pencher la balance de son côté. Le premier se déroule le mardi 16 août, lorsque, au terme de plusieurs journées de conciliation entre des personnalités politiques, des membres de la société civile et des représentants religieux, l'opposition annonce une candidature «unique» autour de Jean Ping. À la surprise des médias et des

32. Durant le septennat d'Ali Bongo, plusieurs opposants ont appelé à une révision de la constitution afin de limiter le nombre et la durée des mandats présidentiels. Rappelons que depuis la réforme de 2003, ce mandat est fixé à sept ans renouvelables indéfiniment, et l'élection présidentielle ne comporte qu'un seul tour.

33. S. Jordan, «Ouverture de campagne d'Ali Bongo: plus de 40000 personnes, beaucoup de social, aucune annonce de réforme politique ou économique» [en ligne], Gabonreview, 15 août 2016, $<$ gabonreview.com/blog/ouverture-de-campagne-dali-bongo-plus-de-40-000-personnes-beaucoupde-social-aucune-annonce-de-reforme-politique-economique/ $>$, consulté le 6 décembre 2016.

34. Ces transactions s'inscrivent dans la continuité des esthétiques de l'acquisition et de conversion de la valeur décrites par F. Bernault, «Aesthetics of Acquisition: Gabonese Spectacles and the Transactional Life of Bodies and Things », Comparative Studies in Society and History, vol. 57, $\mathrm{n}^{\circ} 3$, 2015, p. 753-777. 
leaders du PDG, un front uni se construit entre Guy Nzouba Ndama, Casimir Oyé Mba et Jean Ping, front que rejoindra quelques jours plus tard Léon Paul Ngoulakia, cousin germain d'Ali Bongo et ancien secrétaire général du Conseil national de sécurité, et auquel adhéreront de nombreuses associations de la société civile (Brainforest, Femme lève-toi, Dynamique unitaire et Étudiant conscient).

Après les critiques d'une opposition désorganisée, privilégiant les intérêts individuels à la structuration collective et peinant à concevoir un programme solide, la candidature unique inverse la tendance en faveur de l'opposition ${ }^{35}$. Certes, comme beaucoup de leaders de l'opposition, Jean Ping est un ancien membre du système aujourd'hui en rupture de ban. Père de deux enfants avec Pascaline Bongo et étroitement lié à Omar Bongo pendant 25 ans, il n'est pas un opposant historique comme l'était Pierre Mamboundou, mais c'est précisément cette fine connaissance de l'appareil étatique gabonais, des réseaux du pouvoir local et de la diplomatie internationale qui en font le candidat du ralliement et qui dissipent l'inquiétude de l'inconnu que représenterait un acteur politique hors du jeu étatique. Comme en 2009, l'élection de 2016 oppose donc deux héritiers d'Omar Bongo. Mais à la différence d'André Mba Obame, Jean Ping parvient à fédérer différents caciques du régime Bongo à ses côtés, et sa carrure internationale lui assure des soutiens à l'extérieur du Gabon ${ }^{36}$.

À partir de cette annonce d'un front uni, les meetings de l'opposition rallient une foule plus importante que les rassemblements du PDG, sans pour autant offrir de rétributions monétaires. À Nkembo d'abord, l'un des quartiers les plus défavorisés de la capitale, le meeting où le front uni est révélé, le mardi 16 août, voit se succéder au micro des universitaires de renom comme le sociologue Anaclé Bissiélo ainsi que les personnalités du Front uni de l'opposition qui ont retiré leur candidature au profit de Jean Ping, face à une foule amassée dans les rues et sur les toits du quartier.

Le 21 août 2016, dans la province septentrionale, l'intervention de l'exdéputé démissionnaire Bertrand Zibi Abeghé, accueilli en héros, enflamme les foules. Dans son discours en langue fang, il compare André Mba Obame à Moïse, et Jean Ping à l'élu qui guide le peuple jusqu'aux portes de Canaan. Poursuivant la métaphore religieuse, il incite la foule à entonner l'air d'un chant chrétien, dont il transforme le refrain: «Oh, oh, il nous gêne, Ali doit

35. Entre le 16 août et le 30 août 2016, quatre concurrents (sur 13) rallient la candidature de Jean Ping.

36. Voir F. Bernault, «Le Gabon, le pays où il ne se passe jamais rien » [en ligne], The Conversation, 4 septembre 2016, <theconversation.com/le-gabon-le-pays-ou-il-ne-se-passe-jamais-rien-64856>, consulté le 6 décembre 2016. 
Conjoncture

partir au Nigeria ». Les populations du Woleu-Ntem acclament leur leader local et le candidat Jean Ping en affirmant que « 2016 n'est pas 2009 » et que cette fois-ci, personne ne leur volera la victoire.

Comme le montre cet exemple, la campagne présidentielle de 2016 se distingue également de la précédente sur la problématique ethnique et provinciale. En 2009, la question de l'ethnicité avait été instrumentalisée, tantôt en faveur, tantôt au détriment d'André Mba Obame, cible de la formule: «tout sauf un Fang». À l'inverse, en 2016, la question ethnique n'est pas mise en évidence dans les débats, car l'alliance de l'opposition réunit un savant mélange de personnalités originaires du Haut-Ogooué, d'autres appartenant à la communauté fang, cela autour d'un candidat Myènè.

Finalement, le vendredi 26 août 2016, c'est sur le boulevard Triomphal que Jean Ping clôture sa campagne, entouré de quelque 60000 personnes, d'après les organisateurs. Comme le montrent les vidéos d'euphorie et de chants de liesse qui circulent sur la toile, les espaces publics habituellement associés au régime au pouvoir sont ainsi réappropriés et investis au travers des meetings, transformés en "espaces publics liminaires ${ }^{37}$ ", lieux de protestation et de construction d'une possible émancipation. Au-delà de la personnalité de Jean Ping, la candidature unifiée de l'opposition conforte les Gabonais dans l'idée qu'un système de gouvernement en dehors de la famille Bongo est envisageable, peut-être pour la première fois depuis l'élection de 1993 et le ralliement au pouvoir de l'opposant Paul Mba Abessole. Au-delà d'un candidat ou d'un programme, c'est ici l'expression de ce ras-le-bol contre le système Bongo qui réunit les populations dans les meetings de l'opposition.

Dans ce climat de tensions, entre espoir d'un jour nouveau et inquiétude d'un trucage électoral, arrive le jour J et le début des hostilités entourant le scrutin.

\section{SAMEDI 27 AOÛT 2016 ; MOBILISATION CITOYENNE ET MANCEUVRES ÉLECTORALES}

Bien avant les élections, plusieurs éléments de suspicion sont mis en évidence dans la préparation des élections par la Cenap, et d'abord dans le processus d'enrôlement des populations et de révision des listes électorales. Publiée le vendredi 8 juillet 2016, la liste électorale révisée est immédiatement contestée par l'opposition et la société civile. D’après un audit de Mays

37. M. Hilgers, «Contester en contextes semi-autoritaires: espaces publics en Afrique», Alternatives Sud, vol. 17, 2010, p. 205-220. 
Mouissi ${ }^{38}$, plus d'une cinquantaine de localités comporteraient un nombre plus important d'inscrits que d'habitants, allant parfois jusqu'à 22 fois plus d'inscrits que d'habitants comptabilisés lors du recensement national de 2013. À nouveau, la partialité des instances de la Cenap est éprouvée.

En outre, comme le décrira par la suite la mission des observateurs internationaux de l'Union européenne, les cartes d'électeurs tardent à être distribuées aux populations, et certains inscrits ne parviennent pas à les récupérer à temps. À la veille du scrutin, la cour constitutionnelle annonce aussi soudainement la possibilité de voter au moyen de sa seule carte d'assuré social (carte CNAMGS), le droit pour les militaires de voter en dehors des centres dans lesquels ils sont régulièrement inscrits, et l'ouverture de listes additives d'électeurs pour ces militaires, autorisés à voter même lorsqu'ils ne s'étaient pas inscrits sur le fichier électoral lors du recensement ${ }^{39}$. Ces différentes annonces nourrissent la méfiance des Gabonais et l'inquiétude sur un possible passage en force électoral. Avec l'expansion des réseaux sociaux, cette profonde suspicion conduit à de nouvelles formes de mobilisation le jour de l'élection.

Le matin du 27 août, de longues files d'électeurs se pressent devant les bureaux de vote. La Toile s'enflamme dès le matin d'images et de vidéos que les électeurs capturent dans leurs téléphones portables, pour conserver des preuves audiovisuelles des votes et des décomptes. Dans une atmosphère frisant parfois la paranoïa, des tensions éclatent quand des tentatives de fraude sont soupçonnées. Parmi ces images, beaucoup relatent des arrestations ou des molestations d'individus de nationalité étrangère ayant tenté de voter avec de faux documents; des témoins directs accusent des responsables de bureaux de vote de bourrage d'urnes ou de possession de fausses cartes d'électeurs. Une fois le vote accompli, de nombreux électeurs patientent jusqu'à la nuit tombée pour assister au décompte des voix.

À partir de la fermeture des bureaux de vote à 18 heures, le dépouillement débute, dans les arrondissements de Libreville comme à l'étranger. Dès lors, des estimations des résultats et des copies de procès-verbaux se propagent sur la toile, accompagnées parfois d'enregistrements en direct des proclamations publiques. De son côté, l'économiste Mays Mouissi, en partenariat avec deux

38. M. Mouissi, «Gabon: ces curiosités de la liste électorale qui font douter de sa fiabilité » [en ligne], mays-mouissi.com, 7 août 2016, <www.mays-mouissi.com/2016/08/07/gabon-curiosites-de-listeelectorale-douter-de-fiabilite/>, consulté le 6 décembre 2016.

39. Cette décision a été dénoncée comme «violation flagrante de la loi électorale» par l'opposition, et comme une fraude, car elle permettrait aux militaires de s'inscrire sur plusieurs listes additives et de voter plusieurs fois. Voir AFP, «Présidentielle au Gabon: polémique autour du vote des forces de sécurité» [en ligne], Jeune Afrique, 27 août 2016, <www.jeuneafrique.com/depeches/ 352348/politique/presidentielle-gabon-polemique-autour-vote-forces-de-securite/>, consulté le 26 décembre 2016. 
Conjoncture

médias en ligne gabonais, compile des statistiques en temps réel, à partir des résultats que leur transmettent les états-majors de chaque candidat. Les estimations ainsi faites donnent Jean Ping vainqueur, d'abord à $70 \%$ dans la nuit du 27 au 28 août, puis autour de $60 \%$ le dimanche 28 août.

En théorie, la loi électorale définit la Cenap comme seule habilitée à proclamer les résultats définitifs et enjoint aux partis d'attendre la date de proclamation des résultats, dans un délai maximum de 72 heures après l'élection. Mais avant ce délai, les deux principaux candidats annoncent chacun leur victoire, s'affrontant dans un combat de communiqués. C'est le ministre de la Communication et porte-parole d'Ali Bongo qui enfreint en premier le code électoral, en s'exprimant sur les antennes de la chaîne de télévision nationale le samedi 27 août à 23 h 30: «Même si aucun chiffre ne peut et ne doit être avancé à ce stade, nous sommes, au vu des informations qui nous parviennent, en mesure d'affirmer que notre candidat, Ali Bongo Ondimba, remporte la victoire dans notre pays. Nous sommes donc déjà en route pour un second mandat en attendant la confirmation par les instances habilitées ». Moins d'une heure plus tard, l'équipe de Jean Ping réagit en dénonçant des fraudes, mais aussi cette annonce qui déroge à la sérénité du processus électoral. Elle annonce à son tour que les résultats collectés donnent l'opposition gagnante.

Dans les 72 heures qui suivent, une torpeur saisit Libreville: les marchés sont clos et la circulation réduite à son minimum. Des patrouilles de militaires quadrillent la capitale, survolée par des hélicoptères. Malgré cela, dès le dimanche 28 août à 17 heures, une foule emportée par l'annonce de la victoire de Jean Ping dans plusieurs provinces-clés du pays (par son équipe et les sondages mis en ligne) se réunit devant le quartier général de campagne de l'opposition au quartier des Charbonnages. En dépit de la saison sèche, c'est sous une imprévisible pluie battante qu'ils se mettent en marche jusqu'au rond-point de la Démocratie, en brandissant des rameaux au son d'un nouveau slogan visant nommément Ali Bongo et son directeur de cabinet: «Brûle Ali-Accrombessi». Durant quelques heures, ils manifestent leur joie et appellent au respect de leur vote, affrontant les gaz lacrymogènes des policiers. L'annonce des félicitations officielles du Parti socialiste français au candidat de l'opposition nourrit encore un peu plus l'espoir de ceux qui, partisans ou non de Jean Ping, voient dans cette mobilisation électorale massive la première étape de la fin d'un régime familial qui aura duré cinquante ans. Sur les réseaux sociaux, plusieurs statuts affichent le slogan «Demain, un jour nouveau», détournant cette référence cinématographique nationale des années 1970 pour en faire un des slogans de la révolution électorale en cours. Ironie du sort, le film en question relatait de manière hagiographique l'accession au pouvoir du jeune Albert-Bernard Bongo. 
Après une attente interminable, la Cenap annonce finalement que la commission se réunira le mardi 30 août 2016, à partir de 19 heures. D'après les résultats de huit provinces sur neuf, Jean Ping possède une avance considérable de plus de 60000 voix sur son adversaire. Mais les discussions sont bloquées, officiellement en raison de l'absence du procès-verbal de la province du Haut-Ogooué. Région d'origine de la famille Bongo, située à l'Est du Gabon, le Haut-Ogooué est connu pour être le fief du régime PDG. La Cenap se réunit donc seulement le mercredi 31 août, après l'expédition par avion du procès-verbal en question. Dans le courant de la journée, le vicePrésident représentant l'opposition dans la Cenap, Paul-Marie Gondjout, démissionne, dénonçant des fraudes et un passage en force du Président sortant, tandis que trois autres membres de la Cenap (sur huit votants), s'abstiennent. Malgré ces démissions et crises internes, un verdict est finalement rendu à 17 heures par le président de la Cenap, donnant une victoire de 49,8\% pour Ali Bongo, contre $48 \%$ pour Jean Ping (soit un écart de 5594 voix), avec un taux de $99,98 \%$ de participation et $95 \%$ des scrutins en faveur d'Ali Bongo dans le Haut-Ogooué. En coulisses, un haut cadre du PDG confie que même du temps d'Omar Bongo, «on n'avait jamais vu cela ${ }^{40}$ » et doute que la population admette des résultats aussi peu vraisemblables.

Effectivement, dès l'annonce des résultats, les partisans de l'alternance font entendre leur désaccord par une marche pacifique en direction de la Cenap, mais aussi, très vite, par des rassemblements protestataires dans différents quartiers de la capitale et dans d'autres villes du Gabon. Les forces armées usent de bombes lacrymogènes et des tirs à balles réelles sont entendus. L'Assemblée nationale est incendiée, puis le Sénat et les locaux du quotidien pro-gouvernemental L'Union. Les manifestants sont suivis par des pillards qui s'attaquent aux magasins ${ }^{41}$. De Libreville à Oyem et Bitam dans le Nord, en passant par Lambaréné dans le centre, et Port-Gentil sur la côte, l'ensemble du pays devient, dans les trois jours qui suivent, le théâtre d'un soulèvement et d'une répression que le Gabon n'avait pas connus depuis les émeutes de 1993.

40. AFP, «Gabon: le président Bongo déclaré réélu, émeutes à Libreville» [en ligne], Le Point, 31 août 2016, <www.lepoint.fr/monde/presidentielle-au-gabon-le-parti-au-pouvoir-demande-des-resultatscredibles-31-08-2016-2064955_24.php >, consulté le 6 décembre 2016.

41. Pour une analyse de «l'économie morale du pillage» surgissant dans ces moments de crise, voir les réflexions de Joseph Tonda, «Le Gabon, prisonnier inconscient de l'impérialisme postcolonial» [en ligne], The conversation, 20 septembre 2016, <theconversation.com/le-gabonprisonnier-inconscient-de-limperialisme-postcolonial-65369>, consulté le 6 décembre 2016; ou A. Aterianus-Owanga et J. Tonda, «Préface. Violence de la vie quotidienne, violence de l'événement», in A. Aterianus-Owanga, M. Mebiame Zomo et J. Tonda (dir.), La violence de la vie quotidienne à Libreville, Louvain-la-neuve, Académia, 2016. 
Conjoncture

\section{«BLACK OUT " ET POURRISSEMENT POST ÉLECTORAL}

À partir du mercredi 31 août 2016, les messageries téléphoniques, les réseaux sociaux et internet sont entièrement coupés sur le territoire national, à l'exception de quelques îlots, dans les quartiers généraux du parti au pouvoir et sur des sites pétroliers. L'accès aux télévisions internationales est également suspendu. Durant trois jours, les manifestations de colère sont réprimées par les forces de l'ordre, qui dispersent les manifestants par des tirs à balles réelles et des jets d'eau bouillante. Ceux qui assistent aux échanges relateront quelques jours plus tard, dans les médias notamment, que les corps des victimes sont emportés par les forces de l'ordre vers des destinations inconnues, ou dans des morgues dont l'accès est barricadé par des milices.

Dans la nuit 31 août, le quartier général des opposants, où s'étaient abrités de nombreux manifestants, est cerné par hélicoptères et pris d'assaut par des militaires, au moyen de bombes lacrymogènes et d'armes lourdes, faisant plusieurs victimes. Par la suite, 26 opposants sont séquestrés durant 36 heures, de nombreux blessés sont emmenés dans les hôpitaux, des dépouilles conduites dans les morgues, et les familles partent à la recherche des disparus. En trois jours, 1000 personnes sont arrêtées par les autorités ${ }^{42}$ et emmenées dans des commissariats de la capitale. D'autres, qui s'étaient manifestés dans les meetings pro-Ping avant la proclamation des résultats sont activement recherchés par la Garde républicaine, et contraints de se cacher. La répression des forces de l'ordre face aux populations désarmées conduit à un calme précaire sur la ville et les populations se terrent à domicile.

Vis-à-vis des médias internationaux, les membres de l'équipe d'Ali Bongo déploient rapidement une stratégie de communication visant à inverser les torts et à dissimuler les morts de la répression. Dans sa tournée sur les chaînes de télévision françaises, le ministre de la Communication du Gabon attribue les violences aux opposants, parlant de «pillards» et d'atteinte à la sûreté de l'État. Outre la coupure des voies numériques de communication, les médias d'opposition sont attaqués et les sites de communication des journaux potentiellement critiques sont hackés.

Dans ces stratégies médiatiques, l'interpellation de la «Françafrique » devient rapidement un élément central des débats. Dénonçant par ce terme la persistance des relations de domination, le plus souvent opaques et intéressées, unissant la France à ses anciennes colonies, la critique vise indistinctement

42. Ces chiffres sont ceux donnés par le ministère de l'Intérieur du Gabon. Voir «Violences post-électorales au Gabon: un millier d'arrestations en une journée» [en ligne], RFI, 1er septembre 2016, <www.rfi.fr/afrique/20160901-gabon-le-quartier-general-jean-ping-pris-assaut-forcessecurite>, consulté le 26 décembre 2016. 
les milieux d'affaires (Total, Bolloré, Areva, Veolia), les dirigeants politiques et quelques individus isolés comme Robert Bourgi ou Pierre Péan. Ce spectre de la Françafrique surgit à la fois dans la mise en garde du parti au pouvoir contre toute ingérence de l'État français dans le processus électoral en cours au Gabon, et, à l'inverse, dans les appels à l'aide venus des rangs de l'opposition et d'une partie de la population, qui demandent à la France d'intervenir pour protéger la population gabonaise de la répression postélectorale, ou a minima de ne pas reconnaître les résultats de l'élection. Dans une interview à un média français, Jean Ping énonce ainsi une vision très répandue au Gabon: «La France a des responsabilités historiques au Gabon. Jusqu'à récemment, c'est toujours Paris qui a choisi les dirigeants gabonais; depuis l'indépendance, on a ainsi eu Léon Mba, Omar Bongo et puis son fils Ali Bongo, que Sarkozy nous a imposé [...]. Le Gabon est l'exemple le plus abouti de la Françafrique. Et jamais le peuple gabonais n'a pu faire entendre sa volonté ${ }^{43}$ ».

Les relations entre le Gabon et la France ont pourtant changé depuis 2009, où Nicolas Sarkozy avait été, il est vrai, le premier chef d'État à adresser ses félicitations à Ali Bongo. Pendant le septennat, plusieurs «affaires » ont empoisonné les relations d'Ali Bongo avec la France, comme la poursuite des enquêtes sur les «biens mal acquis ${ }^{44}$ », ou la mise en examen de son directeur de cabinet Maixent Accrombessi en août 2015, pour détournement de fonds publics et blanchiment d'argent. L'assise économique des relations francogabonaises est également sur le déclin, et si la France reste son premier partenaire à travers les entreprises multinationales présentes sur place, le Gabon diversifie ses relations ${ }^{45}$.

De fait, dans les jours qui suivent l'explosion de la crise, les réactions françaises restent frileuses: comme les États-Unis, l'Union européenne et l'Union africaine, le Quai d'Orsay appelle au recomptage des votes et enjoint aux protagonistes d'épuiser les recours constitutionnels. Mais il remet rapidement la négociation entre les mains de l'Union africaine, qui ajourne elle-même la mission qui avait été programmée sur place. Condamnée pour avoir soutenu Ali Bongo dans l'élection précédente, la France souhaite

43. «J. Ping: "Le Gabon est l'exemple le plus abouti de la Françafrique” », Libération, 29-30 octobre 2016, p. 15-16.

44. Voir notamment l'entretien publié par Politique africaine en 2009 à ce propos; F. Pigeaud, M. Ona Essangui et G. Ngbwa Mintsa, «L'affaire des “biens mal acquis” ?», Politique africaine, n 115, 2009, p. 67-73.

45. C'est au Maroc et à l'Attijariwafa Bank qu'échoit la reprise de la banque Bicig (Banque internationale pour le commerce et l'industrie du Gabon) après le départ de la BNP-Paribas, de même que la reprise de la société privatisée Gabon Télécom en 2008; c'est sur le singapourien Olam qu'Ali Bongo s'appuie depuis 2011 dans son objectif de diversification de l'économie gabonaise. L'exploitation de l'uranium gabonais par Areva est désormais terminée et celle de pétrole est en déclin depuis 1997. 
Conjoncture

apparemment se distancier de toute ingérence dans le scrutin gabonais, quitte à fermer les yeux sur une élection frauduleuse.

Sous la pression de la communauté internationale, Jean Ping dépose le 8 septembre 2016 un recours devant la cour constitutionnelle pour contester les résultats de la province du Haut-Ogooué. Mais peu d'optimisme accompagne ce recours et le scepticisme est de rigueur. La cour constitutionnelle est en effet considérée par l'opposition comme assujettie aux intérêts présidentiels, car dirigée depuis 25 ans par la même Présidente, qui s'avère avoir eu deux enfants avec Omar Bongo. Ali Bongo, lui aussi, demande quelques jours plus tard un recomptage des suffrages obtenus par Jean Ping dans certaines communes.

Après avoir épuisé les 14 jours autorisés pour l'examen des recours, la cour constitutionnelle rend son verdict dans la nuit du vendredi 23 septembre 2016, tandis qu'un important dispositif policier est déployé dans la ville. À 2 heures du matin, dans un tribunal quasiment vide et en l'absence des représentants de l'opposition, la présidente Marie-Madeleine Mborantsuo annonce que le recours de Jean Ping est rejeté, et celui d'Ali Bongo accepté, conduisant à une révision à la baisse des scores de Jean Ping dans l'Estuaire. La victoire d'Ali Bongo est finalement validée, avec 50,66 \% des suffrages.

Le lendemain, tandis que Libreville reste déserte et muette, bien loin des images de liesse qui accompagnent d'habitude les victoires électorales, les félicitations de la communauté internationale au Président réélu se font attendre. En Afrique comme en Europe et aux États-Unis, on «prend acte» de cette réélection, tout en appelant à un retour au calme, au dialogue et à la libération des opposants. Les instances légales et constitutionnelles gabonaises ont tranché, la normalité doit reprendre son cours au Gabon.

Dans cette perspective, le Président réélu appelle au dialogue et annonce «tendre la main» aux opposants, leur offrant la possibilité de rejoindre le gouvernement. L'option proposée rappelle la voie de sortie de crise utilisée après la crise électorale de 1993 lors des Accords de Paris, qui avaient conduit au ralliement de l'opposant Paul Mba Abessole et à son entrée au gouvernement.

Mais cette fois-ci, les principaux opposants et le front uni autour de Jean Ping restent campés sur leurs positions, et la tentative de formation d'un gouvernement d'union nationale échoue. Seul Bruno Ben Moubamba, crédité de $0,53 \%$ des voix, entre au gouvernement. Pour sa part, Jean Ping appelle à la mise en place d'un conseil de la résistance et tente d'organiser des actions de résistance pacifique, comme l'appel à une journée de recueillement national. Les manifestations se poursuivent dans la diaspora, et des requêtes sont déposées auprès de la Cour pénale internationale, autant par le gouvernement que par l'opposition, pour faire la lumière sur les crimes survenus durant la crise électorale. L'annonce quelques semaines après l'élection des 
«sérieux doutes» de la mission de l’Union européenne sur la validité du processus électoral, puis la découverte des écoutes et des menaces du gouvernement gabonais à l'encontre de ces mêmes observateurs européens ${ }^{46}$ viennent troubler encore davantage les relations du gouvernement gabonais avec l'Union européenne, et portent un coup supplémentaire à la légitimité déjà contestée du Président.

Sur le plan national, à peine sorti de la crise électorale, le gouvernement fait face à un blocage de l'économie et à une reprise des grèves, tant dans le secteur de l'enseignement que dans celui du pétrole, où les salariés protestent contre les plans de licenciement, ou celui de la Poste, qui empêche le paiement des fonctionnaires domiciliés dans sa banque à la fin du mois d'octobre 2016. Ali Bongo débute ainsi son nouveau mandat en faisant face à une économie très ralentie et une fronde sociale généralisée dont le parti au pouvoir espère sortir par l'organisation de la Coupe d'Afrique des nations (CAN), prévue au Gabon en janvier-février 2017.

Quelles conclusions tirer de cette chronique du processus électoral? Tout d'abord, que contrairement aux discours qui tendent à enfermer l'Afrique centrale et le Gabon dans une matrice intemporelle, en considérant que les élections de 2016 ne seraient qu'une reproduction de toutes les élections confisquées précédemment, 2016 ne fut pas 2009, ni 2005, 1998 ou 1993. Certes, le jeu de réponses entre confiscation électorale et contestation populaire rappelle d'autres élections tenues précédemment. Mais ces comparaisons éclairent surtout les singularités du moment historique, les transformations des logiques politiques au Gabon depuis la mort d'Omar Bongo, et les orientations qu'a prises la restauration autoritaire sous Ali Bongo, face à l'érosion de ses techniques de domination.

La singularité de l'élection de 2016 repose sur plusieurs points. Tout d'abord, sur la présence d'une opposition et d'une société civile unies autour d'un challenger considéré comme crédible du fait de sa connaissance des mécanismes du régime Bongo et de son expérience diplomatique. Ensuite, une génération de jeunes Gabonais nés durant les transitions démocratiques, inspirés par les alternances démocratiques accomplies au Sénégal et au Burkina Faso, et portés, entre autres, par l'influence de certains leaders issus du monde artistique, a permis une mobilisation sans précédent, avant et après

46. Le Journal du Dimanche révèle le 2 octobre 2016 que les observateurs de l'Union européenne ont été mis sous écoute et espionnés durant toute la période de l'élection. Voir «Watergate au Gabon: ce que révèlent les écoutes ordonnées par Bongo » [en ligne], le JDD.fr, 2 octobre 2016, <www.lejdd. $\mathrm{fr} /$ International/Afrique/Watergate-au-Gabon-ce-que-revelent-les-ecoutes-ordonnees-parBongo-814378>, consulté le 6 décembre 2016. 
Conjoncture

l'élection. Enfin, l'issue du contentieux révèle les fissures d'une Françafrique en déclin, démantelée tant par les nouveaux partenaires économiques du Gabon que par les prises de distance de l'État français.

Si l'architecture administrative et juridictionnelle structurant le vote au Gabon a rendu possible la réélection d'Ali Bongo, cette chronique révèle finalement la distance existant entre succès électoral et légitimité politique. En panne de crédibilité dans son propre parti, abandonné par différents piliers qui assuraient la représentativité ethnique et territoriale à l'intérieur du gouvernement, Ali Bongo fait face à une évidente défiance de la société civile et de la population gabonaise, et se voit, au terme de l'élection, isolé sur le plan international.

En 1971, Georges Balandier ${ }^{47}$ notait que la «libération est d'abord libération dans l'imaginaire», une formule reprise par différents chercheurs s'attachant à comprendre les mécanismes d'émancipation et de subversion inventés dans des contextes autoritaires ${ }^{48}$. Certes, ici aussi, les possibilités de libération ou de renversement réel d'un régime contrôlant l'ensemble des ressources économiques, des instances électorales et des forces de défense et de sécurité se sont avérées limitées. Mais à la différence des précédentes élections, les élans portés par la société civile, par une opposition unifiée, et par une nouvelle génération de Gabonais ont permis, durant quelques mois, d'imaginer la possibilité d'un «jour nouveau». Ils ont conduit à réinventer des espaces de protestation politique - au travers de chansons contestataires, de l'usage des réseaux sociaux, d'une réappropriation de l'espace public -, et à structurer la mobilisation pour l'alternance en reliant les acteurs politiques, la société civile, et les Gabonais de la diaspora

Alice Aterianus-Owanga Institut français d'Afrique du Sud (Ifas), Johannesburg Fondation de la Maison des sciences de l'homme (FMSH)

Mathilde Debain

Université Paris 1 Panthéon-Sorbonne Imaf (Institut des mondes africains)

47. G. Balandier, Sens et puissance, Paris, PUF, 1971, p. 163. Rappelons que Georges Balandier fut parmi les premiers et les rares chercheurs à publier des analyses de sciences sociales sur les dynamiques sociales du Gabon contemporain, avant même les indépendances. G. Balandier, Sociologie des Brazzavilles noires, Paris, Armand Colin, 1955.

48. M. Hilgers, «Contester en contexte...», art. cité, p. 216. 
«Demain, un jour nouveau? » Un renversement électoral confisqué au Gabon

\section{Abstract}

\section{"Tomorrow, Another Day?"}

\section{A Confiscated Electoral Overthrow in Gabon}

Written as a chronic, this paper recounts the forces and circumstances that led, during the presidential election of August 2016, to an electoral mobilisation in favour of a change in power, and to its confiscation. It describes in what way the election of 2016 has been different from the previous elections: it shows that the mechanisms of consent used by the system have been eroded, and that a many-sided protest has emerged, contrasting with the logic of passive consent that was considered before as a feature of power relations in Gabon. It shows that if the possibilities of effective overthrow of the regime have been limited, the impetus of civil society, of a unified opposition and of a new generation of Gabonese have allowed, during a few months, to imagine the possibility of "another day" and to invent new spaces of political protest. 